\title{
Bio Scaffolds
}

\author{
N. Alima ${ }^{1(\otimes)}$, R. Snooks ${ }^{1(\otimes)}$, and J. McCormack ${ }^{2(\otimes)}$
}

${ }^{1}$ School of Architecture and Urban Design, RMIT University, Melbourne 3000, Australia

\{natalie.alima, roland. snooks\} @rmit. edu.au

2 Sensi Lab, Monash University, Melbourne 3000, Australia

Jon. McCormack@monash.edu

\begin{abstract}
Bio Scaffolds' explores a series of design tectonics that emerge from a co-creation between human, machine and natural intelligences. This research establishes an integral connection between form and materiality by enabling biological materials to become a co-creator within the design and fabrication process. In this research paper, we explore a hybrid between architectural aesthetics and biological agency by choreographing natural growth through form. 'Bio Scaffolds' explores a series of 3D printed biodegradable scaffolds that orchestrate both Mycelia growth and degradation through form. A robotic arm is introduced into the system that can respond to the organism's natural behavior by injecting additional Mycelium culture into a series of sacrificial frameworks. Equipped with computer vision systems, feedback controls, scanning processes and a multi-functional endeffector, the machine tends to nature by reacting to its patterns of growth, moisture, and color variation. Using this cybernetic intelligence, developed between human, machine, and Mycelium, our intention is to generate unexpected structural and morphological forms that are represented via a series of 3D printed Mycelium enclosures. 'Bio Scaffolds' explores an interplay between biological and computational complexity through non anthropocentric micro habitats.
\end{abstract}

Keywords: Mycelium $\cdot$ Feedback systems $\cdot$ Material agency $\cdot$ Adaptive fabrication

\section{Introduction}

In this paper, we present a novel cybernetic relationship entwining robotics and Mycelium growth. Through an adaptive feedback system of biological and computational agencies we explore the robotic infusion of Mycelium into biodegradable scaffolds. To orchestrate natural growth and decay over time, we posit a strategy where the degradation of form is catalyzed by robotic interaction between material, natural and computational agency. Our aim is to develop design techniques where biological materials influence the robot's movements and thus become a co-creator within the design process. We therefore examine the design tectonics that occur when enabling nature to co-direct the construction of architectural form. The methodology presented here draws from the adaptive processes of biology within a broader ambition to consider how our buildings can grow, adapt, self-repair and biodegrade. As László Moholy-Nagy stated, 


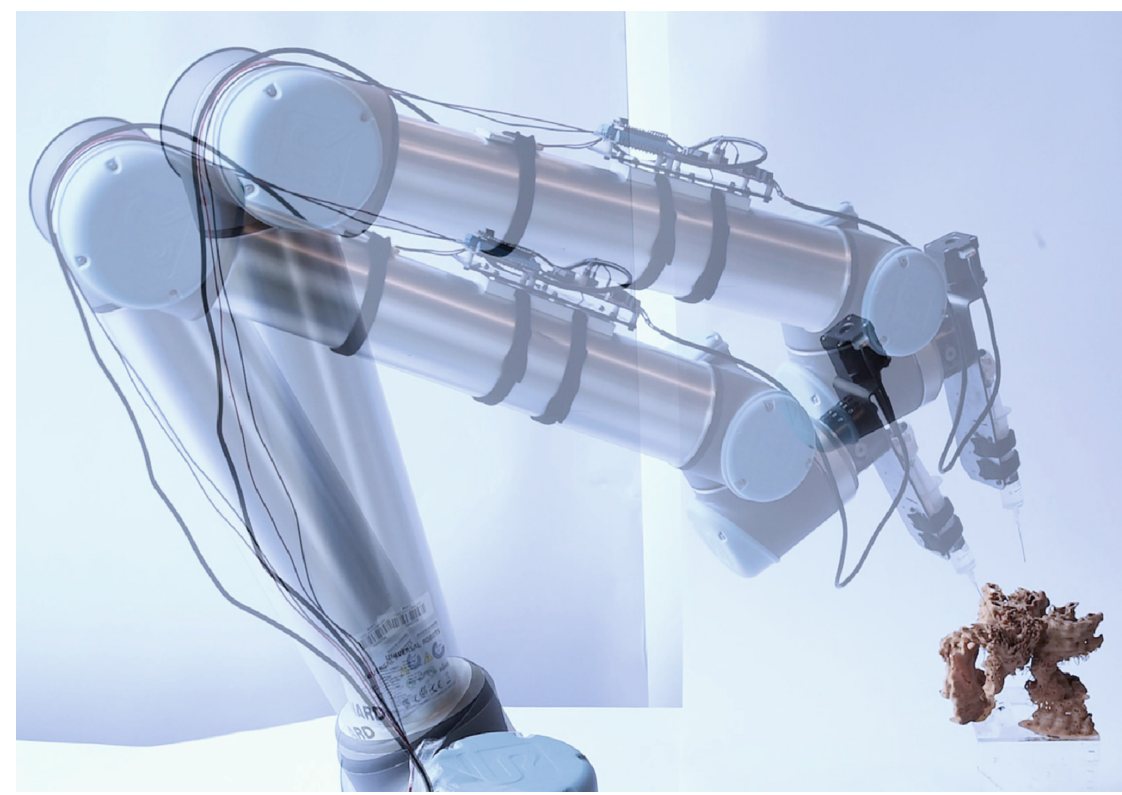

Fig. 1. Universal Robot injecting mycelium liquid culture into 3D printed biodegradable scaffolds.

"architecture will only be brought to its fullest realization when the deepest knowledge of biological life is available" [2]. Within this context, we examine natural materials and demonstrate new relationships between form and environment to speculate on their architectural potential. Our research is therefore interested in the biological process of formation and ways in which it can contribute to design. We encourage other designers to rethink current relationships with nature by enabling a negotiation between biological growth and architectural intention. To demonstrate such a dialogue, here we present BioScaffolds: a sustainable approach and demonstration of novel feedback strategies for architects working with living materials (Fig. 1).

The integration of biological systems within the architectural design and construction processes has a significant lineage and historical precedence. Many disciplines, from engineering through to design and computer science have drawn on natural systems and processes as a rich source of design inspiration. Common methodologies of incorporating nature into design include biomimetics, biophilia and sustainability. Often these methodologies result in a mimicry of nature's forms or its simple application onto existing building structures, without engaging with the agency of material throughout the design process. In this paper we argue that working with nature requires a radical shift toward a new era in which nature is incorporated in both the design and construction processes [11]. An era in which natural growth becomes the catalyst for robotic intervention, 3D printing and computational design.

Within the innovative field of design, Mycelium is increasingly being used for the fabrication of products and buildings. Mycelium is the vegetative part of a fungus bacterial colony and is characterised by mass branching of growth [16]. Mycelium's long, 
branching filaments, known as hyphae are collections of one or more cells surrounded by a cellular wall [3]. Mycelium is often referred to as 'the web of life' as it plays an important role in the decomposition of plant material and pollutants, making them important ecosystem engineers. As Paul Stamets asserted, "I see the mycelium as the Earth's natural Internet, a consciousness with which we might be able to communicate" [4]. We too view mycelium as an integral part of our planet and have therefore become fascinated in enhancing our relationship to nature through design; specially through the creation of form and robotic orchestration.

Today, mycelium is increasingly being used in the fabrication of products and sutainable solutions to building materials. Mycelium's chemical characteristics include, its ability to remove toxins from water, act as a natural binder, act as a good insulator and a moldable and biodegradable substrate [5]. Due to its proven compressive abilities, projects such as Philippe Block's Mycelium Tree demonstrate structural use of the materials, despite its modest compressive capacity of around 30 psi [16]. Designers including Phill Ross have demonstrated that mycelium can be grown and transformed into building blocks of different shapes that are $100 \%$ organic and compostable [7]. Showcased in David Benjamins HiFi tower, mycelium bricks where fabricated in order to showcase sustainable solutions to our design and construction industry. Inserting a Mycelium mixture within a mold, it hardens over time, taking the shape of the desired form. It is then dried to become inactive and no longer a living material [8]. Companies such as 'Myco Composite' are currently utilizing the material as an agricultural bio-product for packaging by moulding the material into predetermined forms to be utlised for human purposes [6].

While Mycelium is increasingly being used in the field of architecture and design, its application is generally subservient to a priori form. What has yet to be explored within the field of design is utilising Mycelium within its live state and exploring its complex patterns of growth. We are therefore interested in the organism's ability to consume and eventually decompose an organic substrate, in order to receive its nutritious properties [8]. This chemical reaction occurs as Mycelium grows by releasing enzymes from the hyphal tips to absorb and digest the surrounding nutrients. As a result Fungi attain energy from their surroundings by branching out and building filamentous Mycelial networks [16]. Due to the material's ability to biodegrade through a range of substances in order to receive its nutrients and fibrous minerals, our original contribution to this field is harnessing the organism in its live state in order to enable natural patterns of growth and agency to contribute to the design and fabrication process. Rather than molding Mycelium to preexisting architectural forms and drying it out [18], this research exploits natural growth, enabling the living material to become a co-creator within the design processes. 'Bio scaffolds' examines Mycelium's ability to biodegrade and destroy architectural forms and host systems. By exploring the potential of natural Mycelium growth within the architectural context, our ambition is to engage nature and material agency within the design process rather than deferring material behavior directly to form. We therefore present a series of design methodologies that emerge from the interaction of living (material) and non-living (machine) behaviors, which currently remains underexplored within the field of Architecture. 'Bio scaffolds' explores the fusion between computational and biological intricacies, resulting in a series of non-anthropocentric Mycelia enclosures. 
Hacking into the degradation rates of natural material, sacrificial frameworks act as architectural habitats for Mycelium to grow and eventually biodegrade. In this research paper, we explore robotic intervention as a tool for maintaining and choreographing's the organisms homeostasis and patterns of growth. In order manipulate natural growth, we explore dynamic feedback system in which machine, nature and computational form are in constant dialogue with one another. As the organism begins to grow within the designed geometry, the robot detects and responds to this data by injecting additional mycelium culture into the sacrificial formworks. This cyclitic process occurs over a seven day period, resulting in a computationally and robotically orchestrated process of natural growth. By fusing technologies and existing processes from the natural, robotic and computational fields, this research invites a multidisciplinary approach to architecture. Architect and academic, Marcos Cruz has said that "A notion of design is emerging whereby interdisciplinary work methodologies is traded between designers, engineers and biologist; giving rise to hybrid techniques, new materials and hitherto unamenable living forms" [10]. In order to truly rethink our relationship with nature, 'Bio Scaffolds' adopts a series of trans-disciplinary techniques from the architectural, medical and scientific disciplines.

\subsection{Medical Bio Scaffold}

This ability to intertwine robotic 3D printing with living materials in the fabrication of biodegradable forms was originally derived from the medical bio-scaffold. Our research draws on techniques from the field of biomedicine, such as tissue engineering, 3D printed artificial organs and bio-scaffolds. Specifically, we have been investigating the processes of medical bio-scaffolds where cells are implanted in order to adopt the geometry of the scaffold. This process occurs by 3D printing a structure, then seeding it with native cells and proteins to encourage cell adhesion and tissue generation [12]. Being a biocompatible and bioresorbable material [13], the scaffold is designed to degrade over time (see Fig. 2). Our research explores how these characteristics of biodegradability and biocompatibility may occur within an architectural context.

\subsubsection{Mycelium: Bio Scaffold}

Adopting an approach similar to the medical bio-scaffold, our research explores the decomposition process of Mycelium. However, rather than allowing this process of growth and biodegradability to occur naturally and randomly as it would in nature, we conducted a series of experiments to influence and disrupt the Mycelium's existing behavioral characteristics through robotic intervention, form and materiality. Using computer-designed geometries, we direct the growth of mycelia by hacking into its existing patterns of growth through complex, nutrient rich scaffolds. Mycelium excrete enzymes to break down resources in their surroundings and assimilate the nutrients to build up their fungal network [17]. This absorption process occurs when fibrous, organic substrates are fed to the organism including, wood chips, coffee grounds, sawdust, biodegradable plastic, cardboard and paper [17]. With this knowledge we tested the organism's ability to biodegrade a wood plastic composite material, comprised of corn starch and wooden fibres. The organism was attracted to the fibrous properties contained 


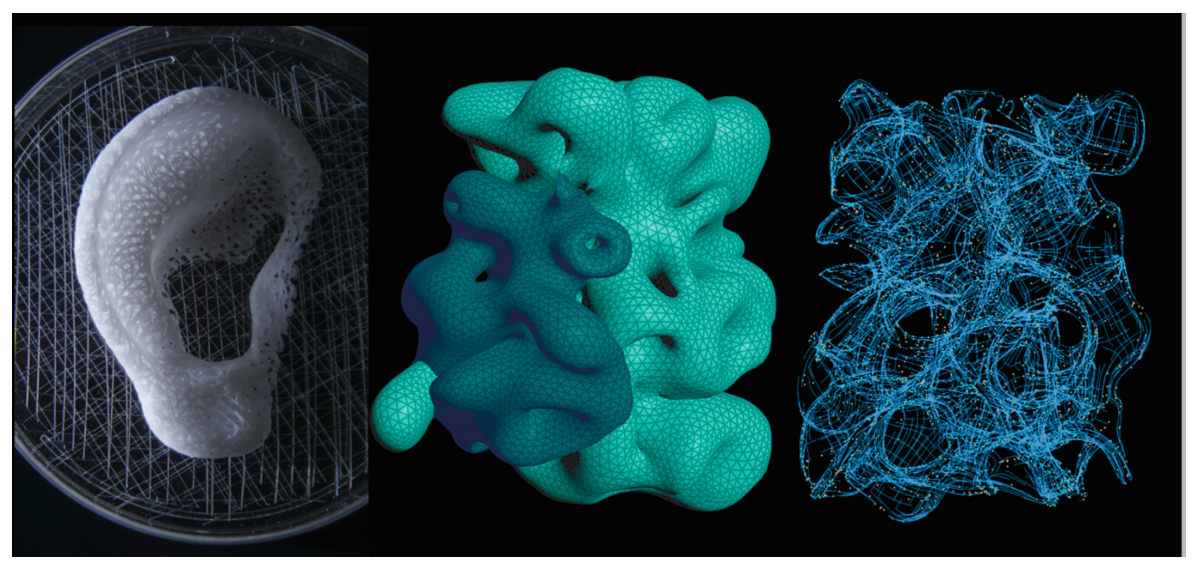

Fig. 2. Far left image (sourced from Computer-Aided Designed, 3-Dimensionally Printed Porous Tissue Bioscaffolds For Craniofacial Soft Tissue Reconstruction Journal) showcases a medical bio scaffold before implantation. Middle image and far right showcases computational forms designed by authors incorporating this time based process of decay

within a wood-based PLA filament, and was therefore capable of growing on scaffolds composed from this material. The following experiments explore 3D printed, biodegradable, nutrient rich scaffolds which manipulate the growth of mycelium through form and materiality. As shown in Fig. 3, this 3D printed geometry demonstrates a successful process of degradation. Mycelium was injected into the wood-based plastic composite forms, eventually degrading the structure and adopting to the set geometry provided. As the Mycelium grows along the scaffold it decomposes and consumes the geometry, replacing the structure with a network of fibrous growth. Growth occurs largely at the tips of the hyphae [5], allowing the Mycelium to spread directly over complex geometric structures as they consume the scaffold.
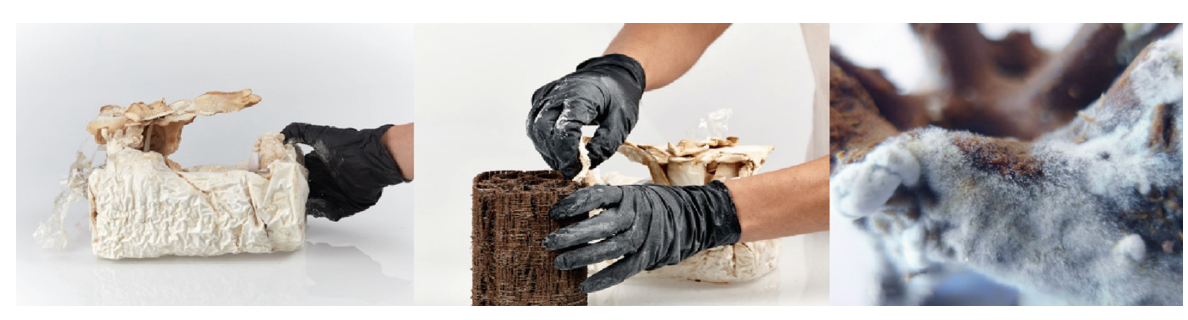

Fig. 3. Far left image showcases growth of organism, middle image showcases bio scaffold printed out of wood based plastic composite material before mycelium inoculation. Far right images showcases mycelium bio degrading wooden scaffold. 


\subsection{Designing with Living Matter}

In order to integrate this unique form finding method with biological growth, our research aims at applying scientific knowledge of Mycelia growth to achieve novel architectural forms. Bio scaffolds entails a layered iterative design process that combines techniques of digital software, fabricated experiments and robotic feedback. Through this fusion of operations, a true hybrid between computation, nature and machine is achieved, as the architect can orchestrate natural growth through form and robotic intervention. Through this nonlinear framework, a singular design process becomes undetectable as the architect's aesthetic and the organism's natural growth become intertwined. Rather than treating nature as a decorative element applied to objects, our approach explores a design and robotic response to Mycelia's natural growth.

Utlising accessible Mycelia cultures, we experimented with the organism and studied its natural growth patterns, in order to manipulate this process through design. This raised the question of how would robotic interference and architectural aesthetic modify the growth of mycelium to achieve a specific growth aesthetic? And what types of geometries would result in the selected biological patterns of growth? Our objective was therefore to study how mycelium propagates over specific forms and surfaces that arise from computational design and robotic fabrication.

During this process, a series of computational experiments were devised to control Mycelium's patterns of growth and behavioral growth characteristics. Each computational form was digitally fabricated and tested by infusing Mycelium culture at key points over the scaffold. This process began with a with a series of computational heterogeneous skins that selectively encourage and hinder the growth of the organism. These features varied in porosity, density and shape and density of internal chambers for the mycelium to seep through. Each geometry was 3D printed using a wood-based PLA filament, composed of approximately $20 \%$ sawdust. Once the scaffold has been fabricated, liquid culture mycelium was robotically injected into it at specific insertion points. We studied the organism's compatibility and rates of degradation, in order to gain a comprehensive understanding of its process of growth and decay. Each experiment was judged and reflected upon in order to examine the ideal forms for successful Mycelia growth. Through these designed experiments, we discovered that Mycelium grows at its most rapid pace along smooth puros surfaces that provide a series of micro valleys for the organism to seep through (shown in Fig. 6). Through this process, we cataloged the inherent qualities of mycelia growth, which included characteristics of branching, bridging and web like strands (see Fig. 4). Each experiment explored a series of intricate weaves and designed obstacles that the Mycelium would grow around, resulting a unique set of growth that would otherwise not occur in nature. Through this interface between machine- and biological-fabricated forms, Mycelia growth therefore became the performative aspect within design, resulting in a series of non-anthropocentric biological enclosures.

However, in order to enable a true co-design between designer and nature, the architect's aesthetic must remain an important contributor to the design process. The preservation of design identity showcases a true duality between nature and architect. Through a series of digitally designed geometries, the designer aims at creating unusual forms that showcase biological material in new and innovative ways. Whilst the geometry has 


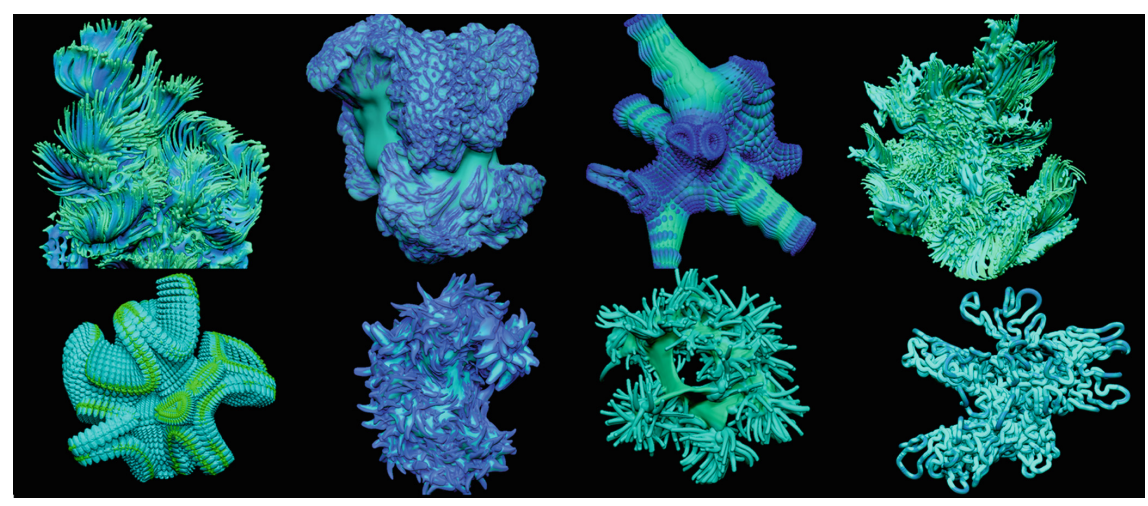

Fig. 4. Computational forms: smooth surfaces designated from mycelium growth

evolved from an understanding of the relationship between digital tools and Mycelium growth, the designer's vocabulary of creating 'mystical creatures' remains an integral part of this process. This co-existence between the architect's design vocabulary and biological agency ultimately exposes a new relationship between computational and biological complexity.

Whilst this research encouraged Mycelia growth through digital tools and robotic fabrication, it also explores an intervention and orchestration of growth. We therefore attempt to also hinder the growth of Mycelium in particular sections of the geometry through a series of articulated forms. Rather than allowing the organism to grow wildly and degrade the entire form, we are interested in a controlled processes, as sections of the geometry are designed to remain or fossilize. In order to achieve this gradience of growth, the scaffolds are comprised of heterogeneous skins what both encourage and hinder life through form. Whilst mycelium latches to smooth surfaces, it is repelled from climbing up vertical antenna lattice systems (Showcased in Fig. 6). We therefore tested a series of scaffold designs that would hinder the growth of mycelium and allow sections of the scaffold geometric integrity to remain. Through a time-based process of decay, we designed fractal components that would remain uneaten by the organism once majority of the scaffold had degraded (Fig. 6).

A series of self-organising generative algorithms were deployed in the design of the scaffolds, including a behavioral design strategy that draws on the logic of swarm intelligence. This multi-agent process, based on the Behavioral Formation approach developed by Roland Snooks, encodes design intention within a population of agents that interact to generate a self-organised design intention and emergent formal assemblages. This algorithmic logic distributes a series of components that create complex topologies and intricate, heterogeneous surface articulation [19].

This contrast between the fibrous intricate details that hinder the growth of mycelium and the smooth valleys that encourage Mycelia growth, enables the designer to orchestrate both the growth and decay of nature. This juxtaposition between designed intricacy and simplicity exposes a contrast between computational and biological complexity. Whilst the smooth surface offers a blank canvas for the Mycelium to spread its growth, 


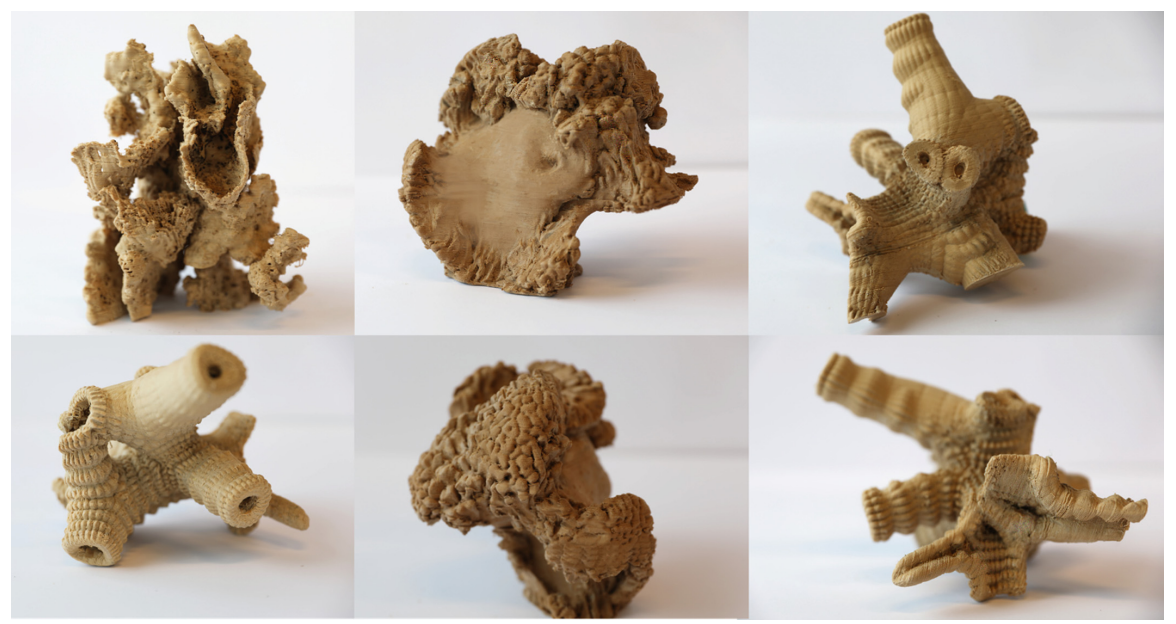

Fig. 5. 3D printed scaffolds from wood based biodegradable plastic infused with corn starch and natural fibers.

the intricate scaffold details enables the architect's aesthetic to be preserved, showcasing a true hybrid between biological and design agencies. Showcased in Fig. 6 nature is working with and against the geometry. Whilst it follows the architectural pathways provided, it also separates from it: affirming its own distinction, independence and individuality. This duality between surface and component enables an ecosystem of interactive geometries, counterbalancing both the biological and computational intricacies.

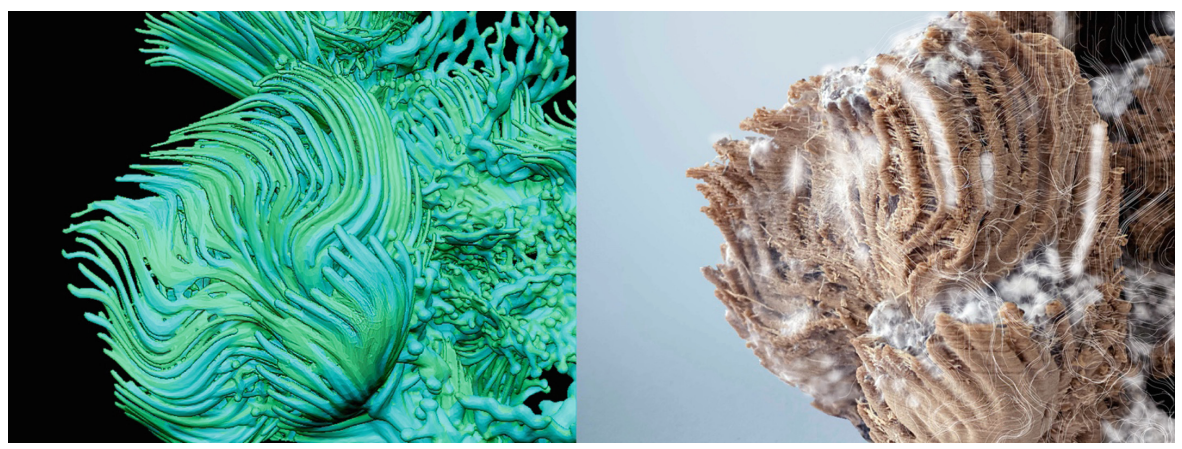

Fig. 6. Image on far left showcases computational model with smooth surface for mycelium to inoculate and designed intricacy that would hinder the growth of mycelium. Middle image showcases this computational model printed out of a wood based bio plastic material. Far left image showcase mycelia growth being orchestrated through these designated forms. 


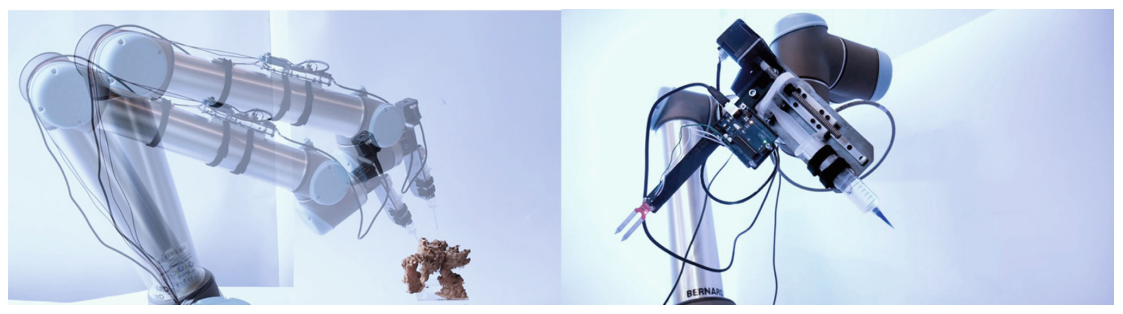

Fig. 7. Universal Robotic customized tool rotating to extract data from mycelium. This tool contains a robotic syringe and Arduino moisture sensors on the opposite end effector.

\subsection{Technical Workflow: Robotic Intervention}

In order to detect and respond to the organism variations of growth, robotic vision systems where implemented that combine form generation, digital fabrication, and material computation into a seamless integrated processes. In addition to orchestrating biological degradation through form, robotic intervention enables us to further intervene on the 'living' with immediate response [1]. As a result, we explored methodologies in which complex fungi behaviors and material data informed robotic feedback. As Mycelium growth may often take place over months or years, robotic intervention seems ideal, since a robot never tires and can act over timescales inconvenient or impossible for human designers. These long-term temporal interventions react to the organism's unpredictability and change over time. During the course of the mycelium's development, the battle and symbiotic tension between machine, organism and form become apparent. As the mycelium begins to spread throughout the sacrificial framework, multiple computational behaviors mutually negotiate to orchestrate the organism's growth. As a result, the robot detects and responds to the organism's properties, including variations in colour, and moisture qualities and responds through systematic feedback loops.

Mycelium, similar to some plants, displays distinct signs when in a flourishing live state in contrast to its dead, dried out state. Visually, these signs include its colour and its moisture content. According to Mitchell P. Jones if the organism is dying, it will display signs of a brown exterior with minimal moisture [8]. In order to keep the organism alive, it requires moisture that maintains its white fibrous exterior. Consequently, we have developed an approach where colour, moisture data and patterns of growth are extracted from the mycelium through robotically controlled scanning and sampling, which then influences the robot's behaviour. The robot reads and responds to this data establishing a symbiotic feedback system where fabrication techniques, robotic intervention and organic development all contribute to the overall design process. Our initial experiments, which hack into nature via robotic intervention, began examining Mycelium's patterns of growth and its ability to biodegrade through a range of complex scaffolds.

The technical components of this research include a computer vision system, 3D scanning process, sensing, and feedback control, 3D printing, and robotic infusion of liquid culture, have been included in the co-creation process between human, machine, and mycelium intelligence. Equipped with customised sensors that track the organism moisture qualities, patterns of growth and colour variation, the technical workflow for Bio 
Scaffold involves a feedback system between the robot, customised tools, material behavior, the vision system and computational form. This series of design experiments used a Universal Robot UR10 controlled by Grasshopper, and 3D printed wood-plastic composite scaffolds, an Arduino moisture sensor, webcam, a customised multi-tool including robotic syringe. These techniques were combined into a single end-effector to scan, read and respond to Mycelium growth in a unified way (see Fig. 7).

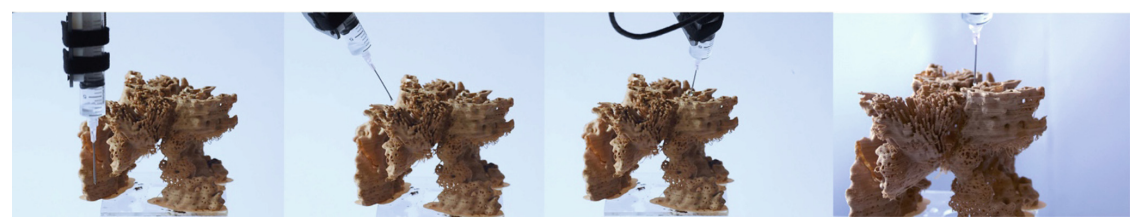

Fig. 8. Technical workflow showcasing the robot scanning, extracting and computationally recognizing this data from the organism

\subsubsection{Feedback System: Computation, Robotic and Material Agencies}

In order to maintain the mycelium's homeostasis, the UR robot operated on the organism over an extended period of time, a process which we refer to as 'slow-botics'. This long-term intervention offers an alternative to the common conceptions of robotics that concentrate on speed and efficiency. Over a 7-day period, the robot waits patiently to receive new data and then acts accordingly [9] (see Fig. 8). Rather than treating the robot and organism as two separate mediums, the robot remains a permanent fixture within in this system, where the behavior of organism and machine support growth and coexistence. The following experiments demonstrate processes in which the robot interpreted data provided by the organism, by identifying the contrasting living conditions of the material, quality of life and areas of nourished or dying mycelium. This feedback system occurred through the following process (see Fig. 9).

\section{Process:}

1. Robotically injecting a liquified culture of mycelium into the scaffold. This step is computationally programed to begin the sensation process.

2. The robot patiently awaits to detect any visual changes as the mycelium begins to degrade throughout the scaffold.

3. Through a process of color detection, The robot begins to continuously scan individual areas of the scaffold to detect properties of white fungi life

4. Once the robot has detected life, a process of edge detection occurs which traces around the identified patterns of growth. This provides the procedure with further parameters, ensuring that the mycelium isn't deposited uncontrollably.

5. The robot responds to these detected growth outlines by probing the designated area of the scaffold with an Arduino moister sensor to extract the mycelium's data (see description of code bellow) This data represents the mycelium's moisture levels and determines whether the medium is living or in a dried out a dead state. 


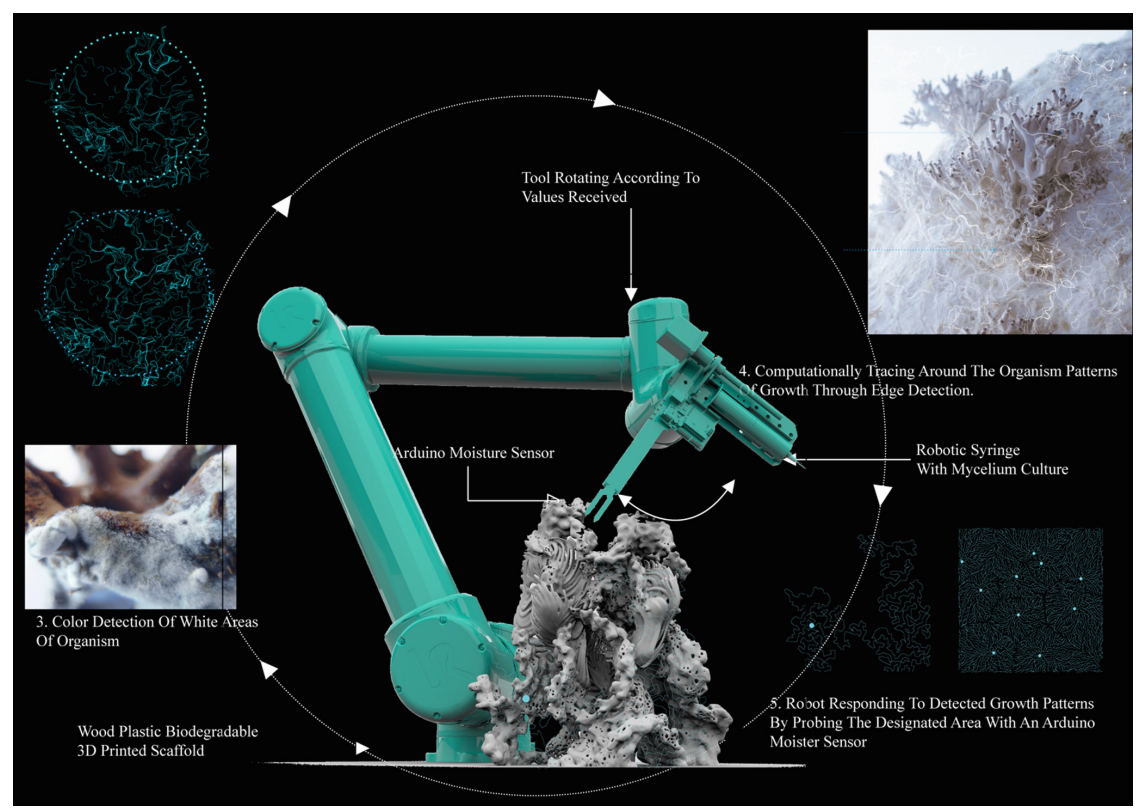

Fig. 9. Technical cyclitic feedback workflow showcasing the robot scanning, extracting and computationally recognizing this data from the organism

6. According to the data received from the moisture sensor, a set of computational rules and restraints are implemented. The algorithm instructed the robot how to respond to the organism. (see computational Logic bellow) If the robot detected that the area was lacking in nutrients and therefore 'barren' it would respond by rotating the singular tool, infusing $30 \mathrm{ml}$ of mycelium. In contrast, if the robot extracted 'living' data and was therefore in a nourished moist state, it would 'do nothing' and proceed to take the next value of its neighboring cell.

During the scanning process, an algorithm is implemented instructing the robot how to react.

Logic:

If value received was $0>50$ then infuse $30 \mathrm{ml}$ of Mycelium

This indicated that the mycelium was extremely dry and was in need of additional moisture.

If value received was $50>80$ then infuse $10 \mathrm{ml}$ of Mycelium

This indicated that the mycelium was in a stable condition and required minimal moisture to maintain this condition.

If value received was $80>120$ then do nothing.

This indicated that the mycelium was extremely moist, do not deposit additional moisture in order to prevent drowning the organism and bacteria. 

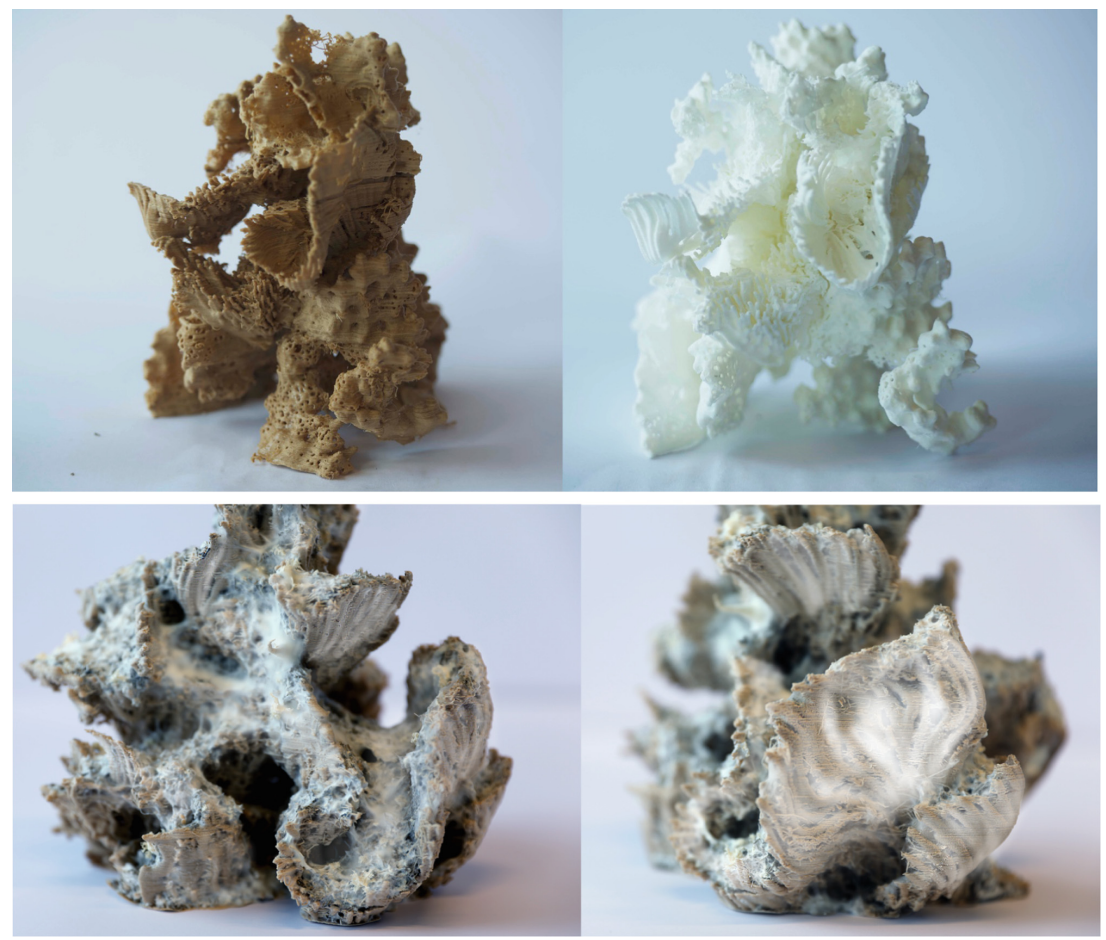

Fig. 10. Results showcasing the orchestration of mycelia growth through form and robotic intervention.

\section{Conclusion}

Resulting from this symbiotic feedback system and form finding process, this research explores the ability to orchestrate natural growth through robotic intervention and Architectural form. Through this time-based process of growth and decay, Mycelia growth is showcased in new and innovative ways that would otherwise not occur in nature. Designing through the interaction of natural systems and computational behaviors creates a complex feedback process that privileges volatility and the unknown. By researching through design, we examined various types of forms which encouraged biological growth. Demonstrated in (Fig. 10) skins that contain dense pours areas, enable the organism to seep through the built framework and degrade it at rapid speed. Similarly, geometries that where based on a branching system, encouraged the organism to extend its patterns of growth along the designated surface area. This research has established an approach where the interaction of these two domains - the physical and the digital, have the potential to generate new and unexpected structural and morphological formations. The interaction of these agencies offers the potential of creating new architectonic approaches, or as Philip Glass states, "A new language requires a new technique" [15]. In this paper we have demonstrated an approach to growing Mycelium through a set of hybrid digital and biological behaviors that interact with computationally generated scaffolds. We are interested in exploring how computational and material agency may 
work together through robotics feedback systems. 'Bio scaffolds' examines the design tectonics and adaptive living forms that may emerge from this process. Through this integration of a unique form finding technique with biological growth, this research aims at merging robotic technologies with living matter until the two become indistinguishable. Whilst the Mycelium enclosures presented are not yet architectural buildings or at architectural scale, they represent progress towards a developed system that engages with materials and addresses sustainability within Architecture.

\section{References}

1. Wahby, M., et al.: A robot to shape your natural plant: the machine learning approach to model and control bio-hybrid systems. In: Sarto, J., Hondlt, T., Mauter, W. (eds.) Evolutionary Computation Conference, LCS, Kyoto, Japan, pp. 1-8 (2018)

2. Anker, P.: Science in culture: Bauhaus at the zoo. Nature 439, 916 (2006)

3. Stamets, P.: Growing Gourmet and Medical Mushrooms, 3rd edn. Ten Speed Press, New York (2000)

4. Stamets, P.: Mycelium Running: How Mushrooms can Save the World, 1st edn. Random House, Berkley (2017)

5. Xing. Y., Griffith, W., El-Gharabawy, H., Brewer, M.: Testing mycelium bricks as building insulation materials. In: Dorhofer, G., LaMoreaux, J. (eds.) Earth and Environmental Science, p. 121, UK (2017)

6. Nishiyama, M., Jun, K., Kazutoshi, T., Kunio Kanaoka, S.: Director-general of agency of industrial science and technology, Okura Industrial. US Patent 5306550 (1991)

7. Dessi-Olive, J.: Monolithic mycelium: growing vault structures. In: Editor, F., Editor, S. (eds.) 18th International Conference Materials and Technologies, Nairobi Kenya, pp. 1-12 (2019)

8. Jones, M., Daver, F., Huynh, T.: Mycelium composites: a review of engineering characteristics and growth kinetics. J. Bio Nanosci. 11, 241-257 (2017)

9. Snooks, R., Jahn, G.: Closeness: on the relationship of multi-agent algorithms and robotic fabrication. In: Reinhardt, D., Saunders, R., Burry, J. (eds.) Robotic Fabrication in Architecture, Art and Design 2016, pp. 218-229. Springer, Cham (2016). https://doi.org/10.1007/9783-319-26378-6_16

10. Cruz, M.: The Inhabitable Flesh of Architecture, New Routledge, New York (2013)

11. Duro Royo, J., Mogas Soldevilaa, L., Oxman, N.: Physical feedback in fabrication information modeling. In: Media Lab, Mediated Matter Group. eCAADe 2015 - 33rd Annual Conference - REAL TIME: Extending the Reach of Computation (2015)

12. Zhou, J., Lu, L.: Biomimetic structured porogen freeform fabrication system for tissue engineering. In: Pramatarova, L. (ed.) InTech, Philadelphia, USA (2008)

13. Chan, B., Leong, K.: Scaffolding in tissue engineering, general approaches and tissue-specific considerations. Eur Spine J. 17(4), 467-479 (2008)

14. Kwinter, S.: The Computational Fallacy. In: Menges, A., Ahlquist, S. (eds.) Computational Design Thinking: Computation Design Thinking. Wiley, London (2011)

15. Oxman, N.: Material - based design computation. Ph.D., thesis, University of Massachusetts Institute of Technology, Massachusetts USA (2004)

16. Heisel, F., et al.: Design, cultivation and application of load-bearing mycelium components: the mycotree at the 2017 seoul biennale of architecture and urbanism. Int. J. Sustain. Energy Dev. 6(1), 296-303 (2018)

17. Appels, F.V.W., et al.: Fabrication factors influencing mechanical, moisture- and water-related properties of mycelium-based composites. Mater. Des. 161, 64-71 (2019) 
18. Boyce, K.J., Andrianopoulos, A.: Morphogenesis: control of cell types and shape. In: Kües, U., Fischer, R. (eds.) Growth, Differentiation and Sexuality. The Mycota, vol 1. Springer, Berlin (2006). https://doi.org/10.1007/3-540-28135-5_1

19. Snooks, R.: Behavioral formation: multi agent algorithmic design strategies. School of Architecture and Design College of Design and Social Context, RMIT University, Australia (2014)

Open Access This chapter is licensed under the terms of the Creative Commons Attribution 4.0 International License (http://creativecommons.org/licenses/by/4.0/), which permits use, sharing, adaptation, distribution and reproduction in any medium or format, as long as you give appropriate credit to the original author(s) and the source, provide a link to the Creative Commons license and indicate if changes were made.

The images or other third party material in this chapter are included in the chapter's Creative Commons license, unless indicated otherwise in a credit line to the material. If material is not included in the chapter's Creative Commons license and your intended use is not permitted by statutory regulation or exceeds the permitted use, you will need to obtain permission directly from the copyright holder.

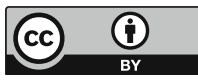

